\title{
Plaque Index and Gingival Index Evaluation in Subjects with Gingivitis after Two Months of Treatment with Two Different Probiotic Food Supply: A Retrospective Study
}

\author{
Michela Zago, Lorenzo Santi, Maria Luisa Caimi, Francesco Cavarra, \\ Vincenzo Rocchetti, and Edoardo Bianco
}

ABSTRACT

Objective: The purpose of this study is to investigate and compare the Gingival Index and the Plaque Index in a population treated with 2 probiotic food supplies (Reuterin Os® and Carioblis $\left.{ }^{\circledR}\right)$ for two months.

Material and Methods: The sample was composed of 92 patients suffering from gingivitis, divided into 4 groups: the first received only causal treatment (group A - control group), the second and the third (group B and $\mathrm{C}$ - test group) took respectively 1 and 2 tablet of probiotic food supplement Ruterin Os ${ }^{\circledR}$ every day for 60 days, the fourth (group D- test group) took 1 tablet of probiotic food supplement Carioblis ${ }^{\circledR}$ every day for 60 days.

The patients were visited every 14 days. Statistical analysis was performed to compare the control group to test groups after 2 months and to compare each index from the beginning to the end of treatment.

Result: The study showed that the use of the probiotics RuterinOs ${ }^{\circledR}$ or Carioblis $₫$ in combination with causal treatment in patients with gingivitis led to a statistically significant improvement in plaque indices (PI) and gingival inflammation indices (GI) compared to the control group subjected only to causal treatment. The analysis of the data also highlighted a better result for patients who took 1 tablet of RuterinOs ${ }^{\circledR}$ every day for 2 months.

Conclusion: The Authors can deduce that the somministration of one daily tablet of ReuterinOs ${ }^{\circledR}$ can bring benefits to oral health.

Keywords: Lactobacillus reuteri, gingivitis, probiotic, plaque.

Published Online: March 26, 2021

ISSN: $2684-4443$

DOI: $10.24018 /$ ejdent.2021.2.2.42

\section{Michela Zago}

Resident

Odontostomatologic clinic, Sant'Andrea Hospital, Vercelli, Italy.

(e-mail: michelazago1994@gmail.com)

Lorenzo Santi

Resident

Odontostomatologic clinic, Sant'Andrea Hospital, Vercelli, Italy.

(e-mail: lore.santi7@gmail.com)

Maria Luisa Caimi

Resident

Odontostomatologic clinic, Sant'Andrea Hospital, Vercelli, Italy.

(e-mail: luisacaimi@alice.it)

Francesco Cavarra

Professor

Department of Clinical Sciences, University of Eastern Piedmont, Novara, Italy.

(e-mail: francesco.cavarra ${ }^{\circledR}$ hotmail.it)

Vincenzo Rocchetti

Professor

Department of Clinical Sciences, University of Eastern Piedmont, Novara, Italy.

(e-mail: vincenzo.rocchetti@med.unipmn.it) Edoardo Bianco*

Professor

Department of Medicine and Surgery,

University of Milano Bicocca, Monza, Italy.

(e-mail: edoardowhite@gmail.com)

*Corresponding Author

\section{INTRODUCTION}

Gingivitis and periodontitis are historically considered to be among the most important global oral health disorders and their distribution and severity can vary in different parts of the world, from country to country or from region to region. Being able to maintain good oral health thus avoiding that a situation of gingivitis degenerates into periodontitis means preventing or postponing some chronic diseases [1].

Gingivitis is defined as an inflammatory condition affecting the soft tissues surrounding the tooth. It is a direct immune response, in reaction to the microbic plaque present on the dental surface which causes inflammation of the epithelial and connective tissue. It is a reversible condition without consequences if good home oral hygiene and adequate professional oral hygiene are performed [2]-[8].
When the oral biofilm, the main etiological factor of gingivitis, reaches maturity, is established a relationship between the host and the microbic species. The inflammatory by-products, together with the bacterial plaque, endotoxins and metabolic products begin the process of periodontal destruction [9].

The causal treatment proposed for periodontal diseases involves mechanical-instrumental treatment associated with the use of chemical-pharmacological aids and the patient's education and motivation for correct daily home oral hygiene; although this treatment method is commonly accepted and used, research and progress in the medical-scientific field have made possible to use additional methods or therapeutic strategies, including probiotics to inhibit the development of oral biofilm and reduce the cascade of harmful immune and inflammatory reactions [9]-[13]. 
There are currently eight known classes of probiotics: seven from bacteria and one from yeasts. The most common probiotics belong to the generic strains of Lactobacillus and Bifidobacterium [1].

Probiotics can have a medical role as a microbial preparation and a functional role through nutrition. They are therefore provided in four different basic ways:

- as a culture concentrated inside drinks or food;

- in prebiotic fibers;

- in a milk-based food (eg dairy products);

- as cellular concentrates packaged in dietary supplements.

Within the "macro-set" of antibiotics there is a subset of bacteriocins. The latter act selectively against specific bacterial strains or species and appear capable of eliminating or interfering with the growth of other bacteria genetically "close" to the inhibiting bacterium. Furthermore, unlike viruses, are considered safe by the Food and Drug Administration for administration in humans and have therefore gained popularity in research in the field of probiotics [14]-[16].

From the side effects point of view, inhibition between species, obtained through bacterial interference mediated by natural antibiotics such as bacteriocins, is preferable to the use of synthetic antibiotic agents, natural antibiotics cause a minor collateral reduction of non-pathogenic bacteria because the antimicrobial spectrum acts on bacteria in the immediate vicinity; for this reason, bacteriocin-producing bacteria are considered a sensible approach to pathogen control [14].

The action of probiotics in the oral cavity could be similar to that which occurs at the intestinal level. In fact, probiotics, in order to carry out their action, must be able to:

- withstand oral conditions,

- resist environmental defense mechanisms,

- adhere to the saliva-coated surface,

- colonize the mouth,

- proliferate to inhibit oral pathogens [9-17].

The strategy action of probiotics is therefore based on the modifications of the potential pathogenic bacteria present in the biofilm, interfering with the growth and development of periodontal pathogens, replacing pathogenic microorganisms with beneficial bacteria, and preventing colonization by periodontal pathogens [18].

Probiotics are thought to work through a number of different mechanisms including:

- competition with potential pathogens for nutrients or for sites of adhesion with enterocytes,

- degradation of toxins,

- chemical inhibition of pathogenic bacteria:

$>$ lowering the $\mathrm{pH}$,

$>$ producing inhibiting compounds,

$>$ reducing the availability of surfaces for colonization by other bacteria.

- stimulation of the non-specific immune response and modulation of the cellular and humoral immune response through the production of immunoglobulins (IgG), defensins and cytokines and the decrease of metalloproteinases (MMP) [1], [19]-[22].

\section{MATERIAL AND METHODS}

The purpose of this retrospective study was to investigate and compare the effects of probiotics contained in the commercial products ReuterinOs ${ }^{\circledR}$ (Nóos S.r.l., Roma, Italy) and Carioblis ${ }^{\circledR}$ (Omeopiacenza S.r.l, Piacenza, Italy) administered to patients with gingivitis after causal treatment and evaluate the trend in two months of the plaque and bleeding indexes, comparing the data to a control group.

For this study 77 subjects with diagnosis of gingivitis, performed by evaluating the presence of a Plaque Index (PI, Löe and Silness, 1964)> 1.1 were recruited at the Sant'Andrea Hospital (Odontostomatologic Clinic - University of Eastern Piedmont) in Vercelli from january 2017 to january 2018.

The selected patients had to fall within the following inclusion and exclusion criteria:

Inclusion criteria:

- be aged between 18 and 80;

- be affected by gingivitis with indexes of gingival inflammation (PI, Löe and Silness, 1963) GI> 1.1 (moderate gingivitis) affecting permanent dental elements;

- having expressed consent to the use of personal and health data when accessing the hospital.

- presence in the mouth of the following teeth: 1.6-2.6-3.64.6-1.1-2.1-3.1-4.1 (not implants)

- no more than 4 teeth missing.

General exclusion criteria:

- Juvenile spongiotic gingivitis

- Allergic and / or lactose intolerant patients;

- patients with systemic conditions and / or conditions that could interfere with the results of the study, for example immunological disorders, pregnancy, ongoing drug therapy that could influence the signs of gingivitis, diabetes, cardiovascular disease, smokers ( $>20$ die cigarettes), use of oral antiseptics and mouthwashes, taking various antibiotics or probiotics in the 3 months prior to the study.

Specific exclusion criteria for dental elements:

- patients with evidence of neglect or lack of interest in the care of their oral hygiene, identifiable with PI index $>1.9$ (insufficient plaque control), or horizontal mobility of III degree and / or vertical mobility,

- dental migration,

- dental crowding,

- ongoing orthodontic treatment,

- incorrectly devitalized or necrotic elements,

- elements with over-contour restorations (restorations and prosthetic crowns),

- elements in primary or secondary occlusal trauma.

During the first session, Plaque Index (Löe and Silness, 1964) and Gingival Index (GI) (Löe and Silness, 1963) were measured on the mesial, distal, lingual / palatal and vestibular surfaces of the teeth: 1.6-2.6-3.6-4.6-1.1-2.1-3.1-4.1 (first molars and upper and lower central incisors on the right and left).

After the acquisition of consent to treatment and after causal treatment (scaling and root planning), the patient deemed suitable was assigned a randomized protocol: group A (control group) or group B, C, or D (test groups) and a second, a third, a quarter and a fifth appointment was set at the clinic, each 14 days, for the assessment of the health status of the gingival tissues (T0, T1, T2, T3, T4). Each time the PI and GI indices were detected on the same dental elements: 
mesial, distal, lingual / palatal, and vestibular surfaces of 1.62.6-3.6-4.6-1.1-2.1-3.1-4.1 (first molars and upper and lower central incisors right and left). All data was collected anonymously.

After carrying out the causal treatment, each patient included in the study was assigned a different protocol (21 for control group, 20 for test group B, 20 for test group C and 16 for test group D):

- Control group (A): after causal therapy, the patient cleaned the oral cavity daily after meals using a toothbrush and devices for oral interdental hygiene (at least twice a day morning and evening). Protocol A included periodic checks to evaluate the progression of gingivitis only after causal treatment.

- Group B: after causal treatment, the patient cleaned the oral cavity daily after meals using a brush and interdental oral hygiene aids (at least twice a day morning and evening) and took one Reuterin Os ${ }^{\circledR}$ tablet for 60 days, dissolving it inside the oral cavity in the evening after home oral hygiene. After taking ReuterinOs ${ }^{\circledR}$, the patient abstained from smoking, did not take food or drink and did not rinse.

- Group C: the patient after causal therapy cleaned the oral cavity daily after meals using a brush and interdental oral hygiene aids (at least twice a day morning and evening) and took two ReuterinOs ${ }^{\circledR}$ tablets for 60 days, dissolving it inside the oral cavity in the evening after home oral hygiene. After taking ReuterinOs ${ }^{\circledR}$, the patient abstained from smoking, did not take food or drink and did not rinse.

- Group D: after causal therapy, the patient cleaned the oral cavity daily after meals using a brush and interdental oral hygiene aids (at least twice a day morning and evening) and took 1 tablet of Carioblis ${ }^{\circledR}$. After taking Carioblis ${ }^{\circledR}$, the patient abstained from smoking, did not take food or drink and did not perform other rinses.

Participants were prescribed quantities of Reuterin Os ${ }^{\circledR}$ or Carioblis ${ }^{\circledR}$ probiotic food supply equal to use for 60 days. Reuterin $\mathrm{OS} \circledast$ tablets is a food supplement containing the patented association of two strains of lactic ferments: lactobacillus reuteri DSM 17938 and lactobacillus reuteri ATCC PTA 5289. It is useful for making an additional portion of lactobacilli to the diet, containing sucralose which contributes to the maintenance of the tooth mineralization. It is recommended to take 2 tablets a day to be dissolved slowly in the mouth, preferably between meals, as stated by the producer. Carioblis ${ }^{\circledR}$ is a food supplement based on a special probiotic strain, streptococcus salivarius BLISTM M18, useful to prevent the cariogenic processes and the periodontal disorders sustained above all by streptococcus mutans. The probiotic is capable of producing four salivaricins (A, M, 9 and MPS) which oppose the growth of streptococcus mutans and streptoccus sobrinus, the main defendants in the cariogenic processes, and of other bacterial species involved in oral dysbiosis. It also releases dextrinase, an enzyme that reduces plaque formation, and urease, which counteracts the acidity of the oral cavity. It is recommended to be taken in the evening, before going to bed (1 tablet per day).

The gingival measurements were performed using a PCPNC and / or Williams type millimeter probe.

\section{A. Statistical Analysis}

The data obtained were subjected to statistical analysis using Social Science Statistic software (www.socscistatistics.com).

The periodontal PI and gingival indexes GI were analyzed at T4 by comparing the control group to groups B, C, and D. Furthermore, the averages of PI and GI at T4 with respect to T0 were compared within each group by means of $\mathrm{t}$ Student (Table I and II).

The normal distribution of GI and PI values for each patient was analyzed using the Kolmogorov-Smirnov test. At T0 the values are normally distributed for both indices.

\section{RESULTS}

The final sample was made up of 77 patients, 21 for the control group, 20 for group B, 20 for group C and 16 for group D.

The means were compared through the Student $t$ test between PI at T0 and T4 per group of teeth (1.6-2.6-3.6-4.61.1-2.1-3.1-4.1) and between the GI at TO and T4 per group of teeth with Student $t$ test for paired data (graph 1; graph 2).

\section{A. Plaque Index Analysis (PI)}

As regards the PI of group B, the difference between T0 and T4 is statistically significant for $\mathrm{p}<0.05$, with $t=-33.16$.

For the PI of group C, the difference between T0 and T4 is statistically significant for $\mathrm{p}<0.05$, with $t=-21.31$.

For the PI of group D the difference between T0 and T4 is statistically significant for $\mathrm{p}<0.05$, with $t=-26.94$.

For the PI of the control group the difference between T0 and T4 is statistically significant for $\mathrm{p}<0.05$, with $t=-16.06$, demonstrating that even periodontal treatment without subministration of any probiotic is effective in leading to a reduction in the plaque index.

Therefore, the Authors analyzed in detail which treatment was significantly more effective than the others. With Mann - Whitney test for unpaired data without normal distribution, which was not found for PI values for all groups in T4, PI values were compared in T4 in the following pairs:

Control group - group B;

Control group - group C;

Control group - group D;

Group B - group C;

Group B - group D;

Group C - group D.

The difference was statistically significant for all test groups (B, C, D) compared to the control group (A), while the difference within the same test groups was not statistically significant.

\section{B. Gingival Index Analysis (GI)}

As regards the GI of group B, the difference between T0 and T4 is statistically significant for $\mathrm{p}<0.05$, with $t=-35.79$.

For the GI of group C the difference between T0 and T4 is statistically significant for $\mathrm{p}<0.05$, with $t=-29.40$.

For the GI of group D the difference between T0 and T4 is statistically significant for $\mathrm{p}<0.05$, with $t=-26.85$.

For the GI of the control group, the difference between T0 and T4 is statistically significant for $\mathrm{p}<0.05$, with $t=-15.84$, demonstrating that periodontal treatment without 
administration of any probiotic is also effective in leading to a reduction in the index of gingival inflammation.

The Authors then analyzed in detail which treatment was significantly more effective than the others. With the Mann Whitney test for unpaired data without normal distribution, which was not found for the GI values for all groups in T4, the GI values were compared to T4 in the following pairs:

Control group - group B;

Control group - group C;

Control group - group D;

Group B - group C;

Group B - group D;

Group C - group D.

The difference was statistically significant for all test groups (B, C, D) compared to the control group (A), and for group B compared to groups $\mathrm{C}$ and $\mathrm{D}$. The difference between group $\mathrm{C}$ and group $\mathrm{D}$ was not statistically significant.

\begin{tabular}{cccccc}
\multicolumn{7}{c}{ TABLE I: MeAN GI FOR AlL GROUPS } \\
\hline & Mean & Mean GI & Mean & Mean & Mean \\
& GI T0 & T1 & GI T2 & GI T3 & GI T4 \\
\hline Control group & 1.35 & 0.36 & 0.31 & 0.34 & 0.49 \\
Test group B & 1.34 & 0.10 & 0.03 & 0.01 & 0.01 \\
Test group C & 1.30 & 0.16 & 0.11 & 0.07 & 0.07 \\
Test group D & 1.38 & 0.42 & 0.21 & 0.12 & 0.10 \\
\hline
\end{tabular}

\begin{tabular}{cccccc}
\multicolumn{7}{c}{ TABLE II: MEAN PI FOR ALL GROUPS } \\
\hline & Mean PI & Mean & Mean PI & Mean & Mean \\
& T0 & PI T1 & T2 & PI T3 & PI T4 \\
\hline Control group & 1.22 & 0.41 & 0.34 & 0.32 & 0.45 \\
Test group B & 1.23 & 0.34 & 0.23 & 0.15 & 0.11 \\
Test group C & 1.26 & 0.31 & 0.24 & 0.20 & 0.17 \\
Test group D & 1.34 & 0.56 & 0.34 & 0.19 & 0.14 \\
\hline
\end{tabular}

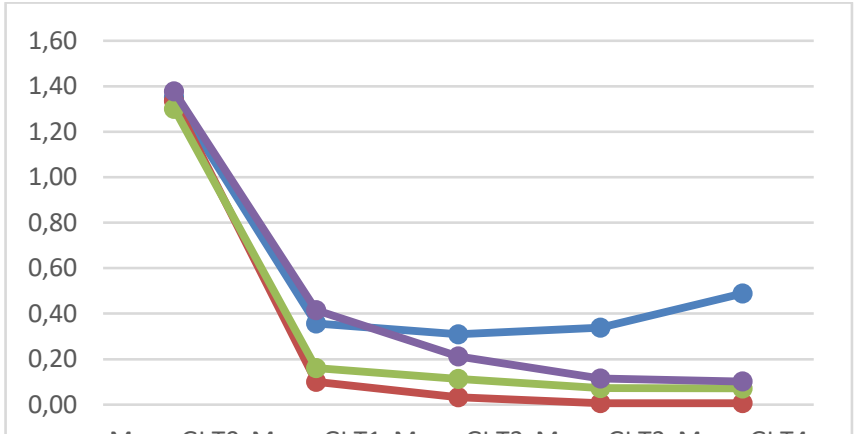

Mean GI T0 Mean GI T1 Mean GI T2 Mean GI T3 Mean GI T4

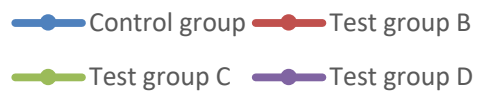

Fig. 1. Gingival index from T0 to T4 for control and test groups.

\section{IV.DISCUSSION}

Taking preventive measures against periodontal diseases as well as their treatment is an additional component to the practice of dental hygiene. The prevalence of periodontal disease is difficult to be accurately defined, but the most recent estimates made by the Center for Disease Control and Prevention (CDC) indicate that $8.51 \%$ of American adults between 20 and 64 years old have periodontal disease. Subsequent studies have determined that more than half of Americans have gingivitis [23]-[26].

There is scientific evidence showing that adding antibiotics or chlorhexidine to periodontal therapy with scaling and root planning improves periodontal health. Despite this, the CDC has defined antibiotic resistance as a growing problem and a direct result of the widespread use of antibiotics, and prolonged use of chlorhexidine can lead to side-effects like loss of taste, taste perturbation and numb feeling of the mouth [25]. In this regard, the role of the dental hygienist comes into play in knowing how to recommend antibiotic therapy only at the right time and in knowing how to consider any other possible strategies such as the administration of probiotics [26], [27].

The data obtained from the observational survey through the data collection of the PI plaque index and GI gingival inflammation in five times T0, T1, T2, T3 and T4 according to protocols A, B, C and D allowed to define whether the use of Reuterin Os ${ }^{\circledR}$ and Carioblis ${ }^{\circledR}$ probiotics, associated with causal therapy, they will or may not benefit the patient's oral health.

Therefore, it was shown that all the test groups (B, C, D) compared to the control group developed a statistically significant improvement in plaque indices (PI) at T4 without significant differences between them. As regards the gingival indexes (GI), however, the test groups show significant improvements compared to the control group but also to each other: group B in fact shows better results than groups $\mathrm{C}$ and $\mathrm{D}$, which instead show no significant differences between them.

As regards the PI values one month after the start of treatment (T2), the PI of the 2 test groups that took ReuterinOs ${ }^{\circledR}$ is lower than the group that took Carioblis ${ }^{\circledR}$ (test group D) and then the control group, which instead are similar to each other. Seemingly, 4 weeks are not a sufficient period of time to notice the differences with the use of Carioblis $\AA$; another interpretation may be the highest starting PI of the test group D. As stated before, in T4 the test groups $(\mathrm{B}, \mathrm{C}$ and $\mathrm{D})$ all have significantly lower average values of $\mathrm{PI}$ $(p<0,05)$ than the control group (group A).

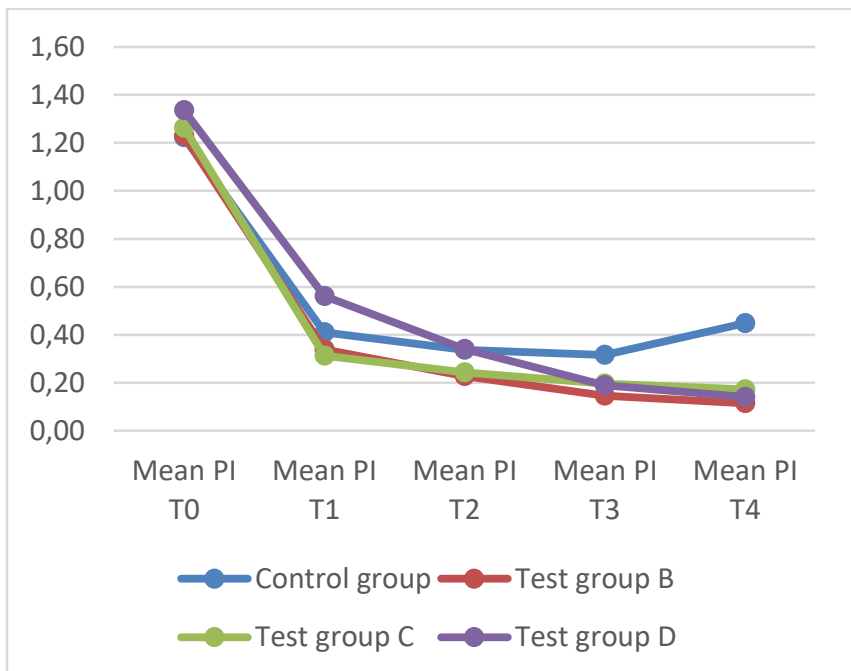

Fig. 2. Plaque index from T0 to T4 for control and test groups.

As regards the GI, after 2 weeks from the start of the treatment (T1) the average values of the control group and of the $\mathrm{D}$ test group are similar between each other and higher than the test groups that took ReuterinOs ${ }^{\circledR}$, testifying a possible slower effect of the probiotic Carioblis ${ }^{\circledR}$ compared to the probiotic ReuterinOs ${ }^{\circledR}$. In $\mathrm{T} 4$ the test groups $(\mathrm{B}, \mathrm{C}$ and D) all have significantly lower average values of GI $(p<0,05)$ 
than the control group (group A).

It can also be noted that at T4 both GI and PI increase for the control group from T3. It should draw attention to the constant need for oral hygiene and the recurrence of plaque if no other intervention is done. This is not noted in the 3 test groups.

Researches conducted on the administration of probiotics show that they cannot cause harmful effects on patients who have an adequate immune system. There are currently eight probiotics considered safe:

1. Lactobacillus reuteri,

2. Lactobacillus casei,

3. Lactobacillus acidophilus,

4. Bifidobacterium lactis,

5. Bifidobacterium longum,

6. Saccharomyces cerevisiae,

7. Saccharomyces cerevisiae,

8. Carnobacterium maltaromaticum.

Any microorganism is capable of causing bacteremia, especially in patients with serious underlying diseases or immunocompromised patients. However, the present literature supports the conclusion that the incidence of Lactobacillus bacteraemia is inconsistent and that all cases where individuals were recorded had other systemic diseases such as diabetes, cardiovascular disease, gastrointestinal disease, malignant tumors, or transplanted organs [19]-[28].

Probiotics therefore represent a new area of research for oral health care. Examination of the close relationship between diet and oral health can potentially be regulated with a variety of different products, such as conventional foods (for the consumption of general populations), dietary supplements (intended not as meals but as oral dietary supplements), medical foods (foods used under medical supervision for patients who need a particular diet in support of a medical condition), medications (to treat, treat, relieve, diagnose diseases) and additives. Recent engineering studies on metabolic expression and protein expression have highlighted the enzymatic and immunomodulatory effect of probiotics: this could allow their use in a greater number of healthy patients and individuals. Furthermore, the implantation and persistence of probiotics are an important requirement for their activity and will have to rely on the synchronized supply of growth factors of the probiotic itself [1], [29]-[31].

\section{CONCLUSIONS}

This research showed that the use of the ReuterinOs ${ }^{\circledR}$ or Carioblis ${ }^{\circledR}$ food supplement probiotic in combination with causal therapy, in patients with gingivitis, led to a statistically significant improvement in plaque indices (PI) and gingival inflammation indices (GI) compared to the control group subjected only to causal therapy.

The analysis of the data also highlighted a better result for patients assigned to group B than for patients assigned to group $\mathrm{C}$ or $\mathrm{D}$ as regards the index of gingival inflammation GI. We can therefore deduce that the administration of only one daily tablet of ReuterinOs ${ }^{\circledR}$ Probiotic is sufficient to feed a recolonization of the oral microflora and to bring benefits to oral health compared to the administration of 1 tablet of Carioblis ${ }^{\circledR}$ or two tablets of ReuterinOs ${ }^{\circledR}$ probiotic food supply, as in the latter case 2 tablets a day could exaggeratedly alter the microflora. Thanks to the evidence demonstrated by the clinical data just illustrated, for daily clinical practice for both dental hygienist and dentist it is recommended to consider a possible coadjutant for periodontal treatment the prescription of 1 tablet per day for 60 days of lactobacillus reuteri DSM 17938 and lactobacillus reuteri ATCC PTA 5289, thus considering probiotics as important support to causal therapy for the maintenance of correct oral hygiene.

Microbiological and medical research in probiotic food supplies is still in their origin and numerous key points need clarification, so further studies are needed.

\section{DECLARATIONS OF INTEREST}

The Authors declare they do not have conflict of interest.

\section{REFERENCES}

[1] Bizzin, B., Pizzo, G., Scapagnini, G. Nuzzo, D., Vasto, S. 2012 Probiotics and Oral Health. Current pharmaceutical design. 18, 5522 5531 .

[2] Grellmann, A.P,. Zanatta, F.B., 2016. Diagnosis of gingivitis: state of the art. Journal of Dentistry \& Oral Disorders 2, 1-8.

[3] Page, R.C. 1986. Gingivitis. Clin Periodontol. 13, 345-59

[4] Verma, D., Jhawar, A., Khinda, N., Anand, D. 2014. Gingival diseases in childhood: A review. Global Journal of Medical Research. 14, 1622.

[5] Singh, B., Singh, R., 2013. Gingivitis- A silent disease. Journal of Dental and Medical Sciences. 6, 30-33.

[6] Kistler, J.O., Booth, V., Bradshaw, D.J., Wade, W.G. 2013. Bacterial community development in experimental gingivitis. PLoS One 8, 1-13.

[7] Bianco, E., Maddalone, M., Porcaro, G., Amosso, E., Baldoni, M., 2019. Treatment of Osteoradionecrosis of the Jaw with Ozone in the Form of Oil-based Gel: 1-year follow-up. J Contemp Dent Pract. 20, 270-276.

[8] Shaw, L., Harjunmaa, U., Doyle, R., Mulewa, S., Charlie, D., Maleta, K., Callard, R., Walker, A.S., Balloux, F., Ashorn, P., Klein, N. 2016. Distinguishing the Signals of Gingivitis and periodontitis in supragingival plaque: a cross-sectional cohort study in Malawi. Appl Environ Microbiol 82, 6057-6067.

[9] Rastogi, P., Saini, H., Dixit, J., Singhal, R. 2011. Probiotics and oral health. National Journal of Maxillofacial Surgery. 2, 6-9.

[10] Segura, V., Ilyina, A., Segura, C.E., Belmares, Y.S. 2015. Etiology and microbiology of periodontal diseases: A Review. African Journal of Microbiology Research 9, 2300-2306.

[11] Barbato, L., Francioni, E., Bianchi, M., Mascitelli, E., Marco, L.B., Tonelli, D.P. 2015. Periodontitis and bone metabolism. Clin Cases Miner Bone Metab. 12, 174-7.

[12] Arunachalam, R., Rajeev, V. 2017. Communicative and Psychologic Management Protocol for Aggressive Periodontitis. World J Dent 8, 67-70.

[13] Caglar, E., Kargul, B., Tanboga, I. 2005. Bacteriotherapy and probiotics' role on oral health. Oral Diseases. 11, 131-137.

[14] Wescombe, P.A., Heng, N.C.K., Burton, J.P., Chilcott C.N., Tagg, J.R. 2009. Streptococcal bacteriocins and the case for Streptococcus salivarius as model oral probiotics. Future Microbiology 4, 819-835.

[15] Gillor, O., Etzion, A., Riley, M.A. 2008. The dual role of bacteriocins as anti- and probiotics. Applied Microbiology and Biotechnology 81, 591-606.

[16] Kalate, F.A., Gholami, L., Alijani, E., Hedayatipanah, M., Kosari, S. 2018. Level of Interleukin-17 in Gingival Crevicular Fluid of Patients with Chronic Periodontitis. World J Dent 9, 495-499.

[17] Stamatova, I., Meurman, H. 2009. Probiotics: Health benefits in the mouth. American Journal of Dentistry. 22, 330-338.

[18] Yanine, N., Araya, I., Brignardello-Petersen, R., Carrascl-Labra, A., Gonzales, A., Preciado, A., Villanueva, J., Sanz, M., Martin, C. 2013. Effects of probiotics in periodontal diseases: a systematic review. Clinical Oral Investigation. 17, 1627-1634.

[19] Meurman, J.H., Stamatova, I. 2007. Probiotics: contributions to oral health. Oral Diseases. 13, 443-451.

[20] Yao, S.G., Fine, J.B. 2014. Probiotics for Bacterial Disease Treatment 
in the Oral Environment. Compendium of continuing education in dentistry. 35, 658-663.

[21] Paineau, D., Carcano, D., Leyer, G., Darqui, S., Alyanakian, M.A., Simoneau, G., Bergmann, J.F., Brassart, D., Bornet, F., Ouwehand, A.C. 2008. Effects of seven potential probiotic strains on specific immune responses in healthy adults: a double-blind, randomized, controlled trial. Immunology and Medical Microbioogy. 53, 107-113.

[22] Doron, S., Gorbach, S.L. 2006. Probiotics: their role in the treatment and prevention of disease. Expert Review of Anti- Infective Therapy. 4, 261-275.

[23] Centers for Disease Control and Prevention 2019. Oral Health Surveillance Report: Trends in Dental Caries and Sealants, Tooth Retention, and Edentulism, United States, 1999-2004 to 2011-2016. Atlanta, GA: Centers for Disease Control and Prevention, US Dept of Health and Human Services.

[24] Bianco, E., Rota, D. 2018 Oral findings in Rett syndrome: An update and review of the literature. Dent Med Probl. 55, 441-445.

[25] Haydari, M., Bardakci, A.G., Koldsland, O.C., Aass, A.M., Sandvik, L., Preu H.R., 2017. Comparing the effect of $0.06 \%-, 0.12 \%$ and $0.2 \%$ Chlorhexidine on plaque, bleeding and side effects in an experimental gingivitis model: a parallel group, double masked randomized clinical trial. BMC Oral Health. 17, 118-125.

[26] Li, Y., Lee, S., Hujoel, P., Su, M., Zhang, W., Kim, J., Zhang, Y.P., DeVizio, W. 2010. Prevalence and severity of gingivitis in American adults. Am J Dent. 23, 9-13.

[27] Mobini, R., Tremaroli, V., Ståhlman, M., Karlsson, F., Levin, M., Ljungberg, M., Sohlin, M., Bertéus Forslund, H., Perkins, R., Bäckhed, F., Jansson, PA. 2017. Metabolic effects of Lactobacillus reuteri DSM 17938 in people with type 2 diabetes: A randomized controlled trial. Diabetes Obes Metab. 19, 579-589.

[28] Bianco, E. 2016. Factors Influencing Immediate Maxillary Dental Implant Placement and Bone Resorption: A Review of the Literature and an Outlook on the Clinical Possibilities Dent Med Probl. 53, 408412.

[29] Raff, A., Hunt, L.C. 2012. Probiotics for Periodontal Health: A Review of the Literature. The Journal of Dental Hygiene. 86, 71-81.

[30] Disale, P.R., Zope, S., Suragimath, G., Varma, A.S., Pisal, A. 2019. Toll-like Receptors: Molecular Microbe Sensors in Periodontium. World J Dent 10, 396-401.

[31] Jamali, Z., Alipour, M., Ebrahimi, S., Aghazadeh, M. 2019. Effect of Halita mouthwash on oral halitosis treatment: A randomized tripleblind clinical trial J Dent Res Dent Clin Dent Prospects. 13, 31-35. 